\title{
Biliary Intraepithelial Neoplasia-3
}

National Cancer Institute

\section{Source}

National Cancer Institute. Biliary Intraepithelial Neoplasia-3. NCI Thesaurus. Code C67493.

Biliary intraepithelial neoplasia characterized by the presence of severe epithelial atypia. 